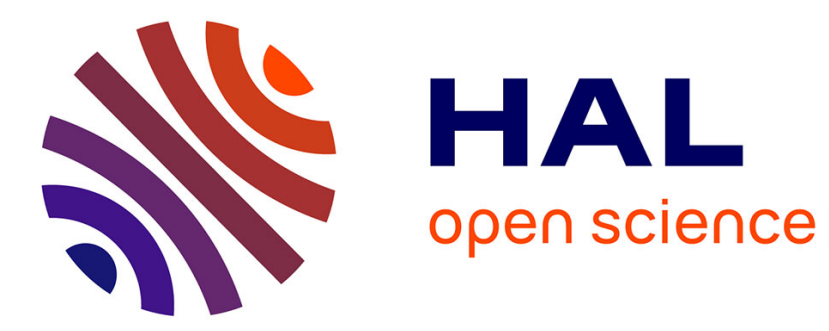

\title{
Théorie et fondements théoriques de la Tétranormalisation
}

Benoît Pigé

\section{To cite this version:}

Benoît Pigé. Théorie et fondements théoriques de la Tétranormalisation. Recherches en sciences de gestion, 2019, N¹31 (2), pp.215. 10.3917/resg.131.0215 . halshs-03425771

\section{HAL Id: halshs-03425771 https://shs.hal.science/halshs-03425771}

Submitted on 11 Nov 2021

HAL is a multi-disciplinary open access archive for the deposit and dissemination of scientific research documents, whether they are published or not. The documents may come from teaching and research institutions in France or abroad, or from public or private research centers.
L'archive ouverte pluridisciplinaire HAL, est destinée au dépôt et à la diffusion de documents scientifiques de niveau recherche, publiés ou non, émanant des établissements d'enseignement et de recherche français ou étrangers, des laboratoires publics ou privés. 
revue Recherches en Sciences de Gestion-Management Sciences-Ciencias de Gestión, n 131 , p. 215 à 240

\title{
Théorie et fondements théoriques de la Tétranormalisation
}

\author{
Benoît Pigé \\ Professeur des Universités \\ CREGO \\ Université de Franche-Comté \\ (France)
}

Le concept de Tétranormalisation est parti de l'observation d'un fait: la situation de blocage à laquelle sont confrontés de nombreux dirigeants d'organisations, non pas en raison de contraintes matérielles ou technologiques mais en raison de contraintes normatives. La réponse naturelle est de tenter de concilier les exigences techniques en recherchant la solution technique qui permet de satisfaire toutes les exigences normatives et donc de faire disparaître le conflit normatif. Une solution alternative consiste à ignorer certaines exigences normatives en effectuant un arbitrage entre le coût du respect des normes et le coût pondéré anticipé du risque de sanction. Une dernière solution existe, qui consiste à repenser le conflit normatif dans un cadre territorial dynamique.

Mots-clés: Tétranormalisation - Institution - Norme - Conflit Territoire.

The concept of Tetranormalization starts from the observation of a fact: the blocking situation faced by many leaders of organizations, not because of material or technological constraints but because of normative constraints. The natural answer is to try to reconcile technical requirements by seeking the technical solution that satisfies all normative requirements and thus eliminates the normative conflict. An alternative solution is to ignore certain normative requirements by making an arbitration between the cost of compliance 
with the standards and the anticipated weighted cost of the sanction risk. A last solution is to rethink the normative conflict in a dynamic territorial context.

Key-words: Tetranormalization - Institution - Standard - Conflict Territory.

El concepto de Tetranormalización comenzó a partir de la observación de un hecho: la situación de bloqueo que enfrentan muchos líderes de organizaciones, no por limitaciones materiales o tecnológicas, sino por restricciones normativas. La respuesta natural es tratar de conciliar los requisitos técnicos buscando la solución técnica que concilie todos los requisitos normativos y, por lo tanto, elimine el conflicto normativo. Una solución alternativa es ignorar ciertos requisitos normativos haciendo un arbitraje entre el costo de cumplimiento de las normas y el costo ponderado anticipado del riesgo de sanción. Una última solución es repensar el conflicto normativo en un contexto territorial dinámico.

Palabras-clave: Tetranormalización - Institución - Norma Conflicto - Territorio.

La Tétranormalisation est un concept forgé initialement par Henri Savall pour désigner l'impact de quatre grandes sources de normes (commerciales, sociales, financières et environnementales) sur le management des organisations (Savall et Zardet, 2005) ${ }^{1}$. Ce concept a permis de fédérer un réseau de chercheurs et a entraîné la réalisation de nombreux travaux de recherche sur les conflits normatifs, qu'il s'agisse de la description de ces conflits, de l'étude de leur origine (Bessire, Cappelletti et Pigé, 2010) ou de propositions pour en sortir (Cappelletti, Pigé et Zardet, 2015).

\footnotetext{
${ }^{1}$ Bien que l'auteur figure parmi les membres fondateurs du réseau Tétranormalisation, les éléments exprimés dans cet article ne reflètent pas nécessairement la pensée des autres membres du réseau Tétranormalisation. Par ailleurs, l'auteur indique n'avoir aucun intérêt économique avec l'Iseor: ni actuel ni passé. Le choix de la revue Recherches en Sciences de Gestion comme support de publication s'explique par le rôle majeur du professeur Henri Savall dans le projet de recherche Tétranormalisation.
} 
Treize ans après la sortie de l'ouvrage de Henri Savall et Véronique Zardet, le paysage de la recherche sur les normes a été profondément modifié. Non seulement le monde politique a reconnu la nécessité de gérer les normes, de limiter leur prolifération normative mais, de plus, les juristes se sont saisis de la problématique normative à travers deux dimensions complémentaires: la prolifération normative (Thibierge, 2014) et la place grandissante des normes dites souples par opposition aux normes dites dures que constituent les textes de loi (Thibierge, 2009).

Qu'apporte donc de spécifique la théorie de la Tétranormalisation et comment se situe-t-elle dans le cadre des théories académiques existantes?

\section{1. - L'objet de la Tétranormalisation}

Le concept de Tétranormalisation est parti de l'observation d'un fait (les conflits normatifs qui paralysent l'activité organisationnelle) et, à partir de ce fait, des analyses de situations ont été réalisées. C'est donc l'observation du terrain (et cela est rappelé régulièrement par le professeur Henri Savall) qui a généré l'émergence d'un concept pour qualifier un état de fait : la situation de blocage à laquelle sont confrontés de nombreux dirigeants d'organisations, non pas en raison de contraintes matérielles ou technologiques mais en raison de contraintes normatives, c'est-à-dire de règles façonnées non par les lois de la nature mais par les sociétés humaines.

\subsection{Le concept de Tétranormalisation}

La Tétranormalisation est un concept difficilement traduisible en langue étrangère en raison de son assemblage d'un préfixe grec Tétra avec le mot français normalisation qui vient du latin norma. Le préfixe Tétra est utilisé en grec pour désigner un objet (ou une réalité) composé de quatre éléments ${ }^{2}$. La racine latine norma renvoie simultanément à l'équerre du charpentier et à la loi (Péron, 2010). Le

\footnotetext{
${ }^{2}$ Exemple : Tetra-logia $=$ tétralogie, ensemble de quatre discours ou pièces de théâtre, d'opéra, de musique.
} 
mot français de règle traduit bien cette double dimension de ce qui permet de mesurer et de ce qui va conditionner le jeu des acteurs. En adoptant le mot normalisation, Henri Savall mettait l'accent non seulement sur le produit final (la norme) mais encore davantage sur le processus qui conduit à la réalisation de la norme et aux effets que celle-ci induit. En effet, la normalisation est le processus qui conduit à rendre normal son objet ${ }^{3}$.

La normalisation consiste donc à rendre un objet, ou le comportement d'une personne $\mathrm{e}^{4}$ ou d'une organisation, conforme à un ensemble de règles (les normes). Mais la normalisation consiste également à édicter les règles qui vont définir la normalité ainsi que les mesures qui pourront être utilisées pour évaluer la conformité de l'objet ou du comportement à la norme.

La difficulté de traduction ne vient pas tant du suffixe Tétra que de l'utilisation du mot français normalisation qui, par rapport au mot anglais voisin, a un sens beaucoup plus large. En effet, si le mot anglais normalization a également le sens de « rendre conforme», les notions de mesures sont rendues par l'usage du mot standardization et les notions économiques de règles du jeu sont appréhendées par le mot institutions.

Mais l'apport déterminant posé par Henri Savall ne vient sans doute pas de l'usage du mot normalisation mais plutôt de l'ajout du préfixe Tétra. En insistant sur la pluralité des normalisations, Henri Savall souligne l'enjeu de la gestion d'une normalisation multiple. S'il y a conflit normatif, c'est parce qu'il y a pluralité de normalisations. La plupart des cas concrets étudiés par les chercheurs recourant à la théorie de la Tétranormalisation font référence à la confrontation entre deux ou trois sources distinctes de normalisation (Tchotourian, 2010; Branellec et Cadet, 2015). Le suffixe Tétra ne renvoie donc pas à la réalité du management concret des normes. Il suffit que deux normes soient en opposition sur un cas concret pour

${ }^{3}$ Dans l'état le plus simple, la normalisation consiste simplement à revenir à l'état habituel. L'anormal est ce qui s'écarte des règles ou des usages établis, voire de la raison ou du bon sens : dictionnaire Lexis Larousse de la langue française (2002).

${ }^{4}$ Pour les comportements humains, le processus de normalisation repose fréquemment sur des formes de stigmatisation des personnes présentant des écarts physiques, psychiques ou comportementaux aux normes habituelles (Goffman, 1963). 
que le dirigeant d'organisation soit confronté à une situation de Tétranormalisation.

Dans la mythologie et dans les textes religieux ou philosophiques, le chiffre quatre renvoie à l'idée d'une universalité (les quatre points cardinaux) mais une universalité marquée par sa différence (les quatre éléments: l'air, l'eau, le feu, la terre). À la différence du nombre douze, qui symbolise une universalité complète et unie (les douze constituent une seule nation, une seule Église, un seul royaume, etc.), le nombre quatre maintient la différence et la séparation entre les quatre éléments. Ces derniers peuvent se rejoindre (d'où les conflits) mais ils ne peuvent se confondre. La théorie de la Tétranormalisation est donc fondée sur le principe que la normalisation, qui est inhérente à tout acte social, contient en ellemême des germes qui interdiront la réalisation d'une unité parfaite. Le mythe de la tour de Babel l'exprime en montrant que la recherche d'une norme unique, d'une mesure unique, d'une règle unique ne peut que conduire à la dispersion (Bessire, 2010).

Alors que la normalisation provoque par elle-même des stratégies complexes de résistance ou de fuite ${ }^{5}$, la Tétranormalisation (c'est-à-dire une pluralité de normalisations provenant des quatre horizons) interdit l'usage de la fuite. En ce sens, la Tétranormalisation présente un caractère apocalyptique marqué, c'est-à-dire qu'elle oblige chaque organisation à s'interroger sur sa raison d'être (ce qu'en droit on dénomme l'objet social). L'obligation de composer avec une pluralité de normes qui interdisent toute échappatoire conduit à une grande diversité de stratégies, certaines visant à une parfaite conformité alors que d'autres les transgressent $^{6}$ au moins partiellement.

Du point de vue des organisations, la Tétranormalisation est un processus mortifère car elle prive le dirigeant d'espace de liberté pour déployer l'énergie de son organisation. Les plus grosses organisations pourront se créer des espaces de liberté (en l'occurrence

\footnotetext{
${ }^{5}$ Que 1'on pourrait paraphraser en citant Oliver Hirschman (1970) : protester ou fuir (voice or exit).

${ }^{6} \mathrm{La}$ fuite correspond à un déplacement hors du champ couvert par la normalisation. La transgression consiste à rester dans le champ de la normalisation mais à ne pas respecter les normes.
} 
des espaces discrétionnaires) en intervenant sur le processus de normalisation, voire même en le pilotant (c'est la raison d'être du lobbying). Les plus petites organisations ne pourront que tenter de gérer les effets de la norme en tâchant de les contourner, de passer sous les seuils d'application ou de contrôle des normes, de minimiser les conséquences des infractions aux normes. D'autres organisations génèrent leurs activités en transgressant résolument certaines normes ${ }^{7}$ tout en s'appuyant sur les normes qui leur sont favorables ${ }^{8}$.

Du point de vue de la société, la Tétranormalisation est un phénomène complexe car chaque acteur est à la fois victime de la Tétranormalisation (oppressé par un système coercitif de normes techniques) et acteur du processus de Tétranormalisation. Parce que la norme permet de répondre à certains de ses besoins ou de ses attentes, le citoyen est demandeur de normes : par exemple sur la qualité de l'eau ou de l'air, sur sa protection en tant qu'employé, client ou fournisseur contre les abus de position dominante, les malversations ou les erreurs.

La Tétranormalisation n'est donc pas un processus maléfique qu'il faudrait combattre par tous les moyens. Elle est un processus naturel consécutif à l'activité humaine. Sa caractéristique, dans les sciences de gestion, est qu'elle conduit à une réduction de l'espace discrétionnaire managérial et que, conduite à sa perfection, elle détruit toute possibilité d'innovation.

\subsection{L'essor de la normalisation technique}

Les romanciers de l'après-guerre craignaient une normalisation idéologique qui n'était que le prolongement des idéologies communiste ou national-socialiste. Dans 1984, la vision de Georges Orwell est celle d'un monde où l'on ne peut plus penser autrement. D'une certaine manière, le cauchemar de 1984 s'est partiellement et temporairement réalisé dans l'Amérique Maccarthyste. Mais l'oppression normative est alors liée à l'unité de la norme qui concentre tous ses efforts pour supprimer toute déviance.

\footnotetext{
${ }^{7}$ Par exemple, l'utilisation d'une main d'œuvre immigrée non déclarée, le travail forcé, l'exploitation sexuelle des femmes ou des enfants, le blanchiment d'argent, la production et la distribution de produits stupéfiants, le financement du terrorisme.

${ }^{8}$ Par exemple, la liberté de circulation des capitaux, des biens et des personnes.
} 
Telle n'est pas la problématique de la Tétranormalisation. Pour la théorie de la Tétranormalisation, l'oppression ne vient pas de l'unité de la norme (qui interdirait toute contestation ou toute divergence d'opinion) mais de la diversité et de l'universalité des normes. Parce que les normes proviennent d'horizons divers et parce qu'elles couvrent l'ensemble des activités humaines, elles aboutissent à un faisceau de contraintes multiples, chacune gérable individuellement, mais impossibles à affronter quand elles sont prises dans leur globalité.

Paradoxalement, la prolifération normative résulte du souci de lutter contre l'hégémonie de l'État. Comme l'a analysé Malcolm Rutherford (1983, p.730), un des apports fondamentaux de John Commons a été la proposition de développer des agences gouvernementales susceptibles d'instituer des règles de comportement ${ }^{9}$. L'originalité de cette proposition tient dans son fondement. Pour John Commons, les agences seraient le pendant des tribunaux. Alors que les tribunaux règlent les conflits ex post, le rôle des agences serait d'édicter des règles de comportement (des normes) ex ante.

La prolifération mondiale des organes de normalisation résulte donc d'un double mouvement: la constitution de la normalisation comme marché avec ses acteurs, ses produits et ses clients $^{10}$; et la réduction des conflits en normalisant les comportements des organisations. La prolifération normative n'est donc pas un processus incompréhensible. Elle répond au désir de limiter la conflictualité entre les acteurs économiques. Parce que les normes vont définir ce qui est acceptable et ce qui ne l'est pas, ce qui doit être fait et ce qui est interdit, elles vont de facto réduire les conflits entre les parties prenantes ${ }^{11}$.

\footnotetext{
${ }^{9}$ Ce mouvement initié aux Etats-Unis s'est traduit en France par l'apparition des diverses Autorités (AMF - Autorité des Marchés Financiers, ANC - Autorité des Normes Comptables, etc.).

${ }^{10}$ La normalisation comptable s'inscrit pleinement dans ce cadre avec les conséquences économiques de la normalisation sur l'activité de conseil et d'audit des grands cabinets comptables.

${ }^{11}$ De la même manière que les règlements de copropriété ou les règlements intérieurs permettent de distinguer précisément ce qui est autorisé ou exigé de ce qui ne l'est pas. Il s'agit donc d'une application concrète des thèses développées par John Commons.
} 
La théorie de la Tétranormalisation traduit cette internalisation des conflits. Parce que la norme permet de prescrire $e x$ ante le comportement souhaité, c'est chaque acteur qui se trouve contraint de devoir gérer en interne les conflits résultant de la pluralité des normes. Au lieu de devoir départager judiciairement ex post les parties belligérantes sur des décisions ou des actes passées, les normes édictent les comportements supposés assurer l'harmonie entre les acteurs.

Cette approche a deux conséquences qui ont été sousestimées et que la théorie de la Tétranormalisation permet de prendre en compte. La première est que la normalisation crée par elle-même des sources de conflits entre acteurs. En l'absence de normalisation, des désagréments locaux pouvaient être résolus par la bonne volonté des parties en présence et par l'intérêt à maintenir des relations harmonieuses pour le développement des collaborations futures. L'existence de normalisations techniques conduit non seulement les acteurs à s'appuyer sur les non-conformités relevées dans le cas de conflits où les acteurs ont subi un préjudice direct, mais elle peut également générer des conflits supplémentaires quand le délit n'est plus la conséquence du non-respect des normes mais la simple nonconformité. Autrement dit, la prolifération normative peut conduire à une multiplication des conflits et donc à une augmentation des besoins judiciaires.

La seconde est que la norme est toujours imparfaite, au sens qu'elle ne peut pas intégrer ex ante tous les cas de figure possibles ${ }^{12}$. La norme peut donc, dans certains cas, conduire à des comportements néfastes pour la société et pour les relations entre les acteurs économiques et sociaux ${ }^{13}$. Parce que la norme est imparfaite, la Tétranormalisation, en réduisant l'espace de liberté du dirigeant, limite l'exercice individuel de l'éthique. Le dirigeant, ou l'employé, n'a plus à s'interroger sur la pertinence éthique de ses choix, il lui

${ }^{12}$ La perfection n'est sans doute pas souhaitable car elle conduirait à l'interdiction de toute liberté. Historiquement, les grandes découvertes et innovations contiennent toujours une part de transgression des normes existantes.

13 Par exemple, la maximisation de l'efficience économique par l'utilisation des méthodes managériales privées peut conduire à une exacerbation des conflits au sein du monde hospitalier (Dupuy et Naro, 2015). 
suffit de considérer que son comportement a été conforme aux multiples normes qui lui étaient imposées ${ }^{14}$.

\subsection{La conformité et la perte de la hiérarchie normative}

Dans l'approche économique classique, la norme a pour objet de corriger une externalité en obligeant les organisations à internaliser les conséquences de leur activité. D'un point de vue abstrait, l'organisation a donc le choix entre poursuivre son activité, en incorporant dans ses processus les conséquences de la norme, ou arrêter son activité. En réalité, les choix de l'organisation se situent sur un continuum entre ces deux points extrêmes. L'organisation peut décider ${ }^{15}$ de respecter tout ou seulement partie de la norme en arguant ${ }^{16}$ des spécificités de son activité ou de son environnement. Théoriquement, le comportement organisationnel signifie que l'organisation choisit (formellement ou informellement) d'internaliser ou non les conséquences de ses processus et de ses actions.

La conformité peut être perçue comme une contrainte pour les organisations qui la subissent ou comme une opportunité pour les organisations qui ont la capacité d'en édicter les règles ou d'influer sur le processus normatif. Dans le premier cas, toute norme apparaît comme une limitation de la capacité d'agir. Dans le second cas, la norme peut faciliter l'action en permettant de mieux contrôler les externalités générées par des concurrents, des clients, des fournisseurs ou, de façon générale, les acteurs susceptibles d'influer sur les processus de l'organisation.

Les organisations qui sont capables de dégager suffisamment de ressources pour les affecter au management de la Tétranormalisation ont non seulement la possibilité d'identifier toutes

\footnotetext{
${ }^{14}$ La plupart des grands scandales organisationnels de ces dernières décennies ne résultent pas tant d'une malhonnêteté intentionnelle de leurs auteurs que d'une absence de regard critique et éthique sur leur conduite, que ce soit l'affaire Enron, la faillite de Lehman Brothers ou même le non-respect des règles internes de la Société Générale par Jérôme Kerviel.

${ }^{15}$ Le processus de décision peut être formel mais, le plus souvent, il est informel et correspond à une compréhension tacite entre les acteurs concernés par le phénomène précis de normalisation.

${ }^{16}$ Sans qu'il ne s'agisse nécessairement d'une argumentation formalisée, elle peut être implicite et sous-jacente aux représentations des divers acteurs.
} 
les contraintes normatives, afin de déceler les interstices dans lesquels une stratégie innovante peut se loger, mais elles ont aussi la capacité d'influer sur la formation des normes dans les domaines qui les affectent le plus. Le lobbying ${ }^{17}$ n'est donc pas uniquement une technique visant à faire entendre (ou prévaloir) son intérêt particulier dans le concert de l'intérêt général. Le lobbying vise aussi à redonner à l'organisation des marges de manœuvre pour lui permettre de trouver des solutions originales et innovantes aux demandes multiples et contradictoires qui lui sont adressées.

Le lobbying présente de sérieux effets collatéraux. D'une part, il limite la prise en compte des intérêts particuliers aux seuls acteurs susceptibles de dégager les ressources nécessaires ${ }^{18}$ mais, en plus, il peut se transformer en outil de défense ou de renforcement des positions acquises. Par exemple, le lobbying ${ }^{19}$ peut aboutir à un renforcement normatif pour interdire l'entrée de nouveaux concurrents sur le domaine d'activité des entreprises existantes, voire pour éliminer un concurrent existant.

À côté de la stratégie de lobbying, les organisations peuvent choisir de s'affranchir du respect des normes en effectuant un arbitrage entre le coût de la sanction et la probabilité d'être sanctionnées par rapport au coût (visible ${ }^{20}$ et caché $^{21}$ ) du respect des normes. Selon le statut des normes, l'absence de conformité présente des conséquences différentes. Dans le cas des normes dures liées au droit pénal (par exemple, les déclarations liées à la lutte contre le

${ }^{17}$ Le lobbying est un mode d'expression de la capacité des acteurs à agir et donc à modifier leur environnement normatif. Cela rejoint le concept d'unité active développé par Perroux (1973).

${ }^{18}$ Ce problème peut être contourné par la création de syndicats représentatifs des intérêts sectoriels d'une profession.

${ }^{19}$ La profession comptable s'est ainsi organisée pour à la fois édicter des normes permettant la défense de la profession (en limitant l'arrivée de nouveaux concurrents dans le domaine de la tenue de comptabilité) et combattre les normes qui lui interdisaient l'accès à des marchés annexes (par exemple le marché des actes juridiques ou des conseils fiscaux). La conquête de parts de marché ne dépend plus alors des seules stratégies individuelles des entreprises mais également de leur capacité de lobbying pour imposer ou éliminer les normes qui encadrent leur activité.

${ }^{20}$ Par exemple, une norme qui impose des dépenses supplémentaires (pour le respect de l'environnement, la protection des employés, la libre concurrence, etc.).

${ }^{21}$ Par exemple, une norme qui empêche le lancement d'un produit ou d'un service innovant ou qui interdit l'accès à un marché (comme c'est le cas pour les pays sous sanctions économiques des Etats-Unis). 
blanchiment d'argent ou le financement du terrorisme), le non-respect des normes peut se traduire par des sanctions non seulement financières mais également personnelles (la mise en cause pénale des dirigeants ou des employés). Dans le cas de normes dites souples (par exemple, le respect de coutumes locales ou le respect des codes d'éthique), l'infraction aux normes peut être perçue comme une simple incivilité.

Pour les juristes, la norme se différencie de la loi par la variété des formes qu'elle peut prendre. Alors que la loi dérive d'une hiérarchie strictement établie : la constitution, la loi, les décrets, les arrêtés, la norme vient s'immiscer de façon horizontale à l'intérieur de ces différents niveaux. Le travail des législateurs est la lutte sans cesse renouvelée pour incorporer dans l'arsenal législatif national (ou supranational, comme l'Union européenne) des normes issues de légitimités diverses (par exemple, les normes comptables internationales qui font l'objet d'une adoption par l'Union Européenne au fur et à mesure de leur édiction). Les menaces du président américain de retirer les Etats-Unis de certains organismes internationaux ${ }^{22}$ découlent du constat que même le pays le plus puissant de la planète n'arrive pas à gérer le flux des normes qui viennent s'intercaler dans son arsenal législatif.

$\mathrm{Du}$ point de vue juridique, le conflit normatif n'est problématique que parce qu'il existe une injonction de respect de la norme et que la norme a force de loi, bien qu'elle n'ait pas forcément été élaborée directement par les pouvoirs législatifs du pays ou de la communauté territoriale. Par exemple, en adoptant les normes IFRS, les pays s'engagent à adopter également les évolutions normatives. Autrement dit, les pays renoncent en partie à leur souveraineté législative et à leur capacité d'arbitrage entre des normes conflictuelles.

De même, dans le domaine des Droits de l'Homme, l'absence d'ordre engendre des contradictions multiples entre droits dotés de la même valeur. L'espace d'interprétation croissant qui en résulte (...) laisse place à une subjectivité parfois dangereuse. La norme éthique

${ }^{22}$ Par exemple, les propos de Donald Trump à l'encontre de l'OMC (Organisation Mondiale du Commerce) en 2018. 
finit par se vider de toute substance au profit de normes techniques (Cadet, 2010, p.152).

Le pouvoir législatif de cohérence de la loi (qui consistait à unifier les règles à respecter par les citoyens au sein d'un pays) disparaît progressivement et chaque personne morale ou physique se trouve devoir arbitrer à son niveau personnel.

\section{2. - Les fondements de la théorie de la Tétranormalisation}

S'il existe un consensus sur l'objet de la Tétranormalisation, les fondements théoriques de la Tétranormalisation demeurent relativement peu explorés. En effet, la théorie de la Tétranormalisation ne nous dit pas grand-chose sur la vision de l'être humain ou de l'humanité qui est sous-jacente. À titre d'exemple, une organisation peut être confrontée à un faisceau de normes contradictoires qui l'empêchent de faire preuve d'innovation, mais cette innovation estelle souhaitable? Au nom de quoi faudrait-il systématiquement promouvoir le développement et l'innovation au détriment de la stabilité et de la conservation des acquis?

\subsection{L'approche socio-économique des organisations}

Alors que l'économie classique s'est développée en dissociant les questions économiques des questions sociales, l'approche socio-économique réintroduit explicitement le lien entre ces deux dimensions en postulant que toute interaction sociale a simultanément une conséquence économique et que tout fait économique renvoie également à une situation sociale.

L'approche socio-économique développée par l'ISEOR ${ }^{23}$ a mis en pratique cette interconnexion en mesurant systématiquement les coûts liés à des dysfonctionnements dans les relations sociales au sein des organisations (absentéisme, conflits hiérarchiques, etc.). C'est donc à partir de la valorisation des coûts cachés liés aux conflits normatifs que la Tétranormalisation a été développée.

\footnotetext{
${ }^{23}$ Institut de socio-économie des entreprises et des organisations.
} 
L'approche initiale de la théorie socio-économique a été lancée dans les années 1930 par des auteurs qui ont essayé de trouver une alternative aux deux modèles dominants: le modèle de l'économie abandonnée aux marchés et le modèle de l'économie administrée par l'État. Dans l'un et l'autre cas, l'économie était considérée comme un objet qui pouvait soit être remis entre les mains d'un opérateur invisible, mais supposé omniscient (le marché), soit entre les mains de l'État (opérateur pleinement visible mais également supposé omniscient à travers les outils de la planification économique).

L'approche socio-économique, développée notamment par François Perroux (Maréchal, 2003), s'appuyait sur la prise en compte des acteurs intermédiaires en partant du postulat que le fondement de toute société et de toute économie, c'est l'Homme ${ }^{24}$. Par conséquent, une théorie socio-économique ne peut trouver des solutions aux problèmes économiques qu'en revenant systématiquement aux valeurs qui fondent l'humanité. De plus, l'analyse économique statique ne suffit pas pour appréhender la réalité des phénomènes. Il convient de les replacer dans une perspective historique en tenant compte de leur trajectoire et des événements qui leur ont donné naissance (Perroux, 1973).

\subsection{La théorie institutionnelle des organisations}

Le concept français de normes ne renvoie pas seulement aux normes techniques mais également aux normes comportementales (Simon, 1945 ; Cyert et March, 1963), culturelles, historiques, aux conventions entre acteurs (Lewis, 1969). Dans les approches académiques, cette dimension de règles du jeu des acteurs est appréhendée par les théories institutionnaliste et néo-institutionnelle.

La conception de la Tétranormalisation est celle d'un conflit qui limite les possibilités d'action de l'organisation, alors que la théorie institutionnelle a une vision plus neutre des institutions. Dans la pensée de John Commons (1931), les institutions (terme anglais que l'on peut traduire par normes en français) peuvent entraver ou faciliter

${ }^{24}$ Avec notamment la théorie du personnalisme développée par Emmanuel Mounier (Mounier, Inyesto et Palacios, 1950). 
l'action individuelle ${ }^{25}$. Leur rôle est donc ambivalent selon les acteurs concernés et les buts poursuivis. Les institutions sont essentielles car elles réalisent le lien entre les actions individuelles et collectives dans la réalisation des transactions. À la différence de la pensée économique classique, où les acteurs individuels se confrontent dans le cadre de règles du jeu supposées naturelles ou imposées par l'État (Rutherford, 1983), dans la pensée de John Commons la confrontation entre les acteurs individuels est encadrée par les institutions qui sont elles-mêmes le résultat du jeu passé des acteurs ${ }^{26}$. Pour Thorstein Veblen (1898), le principal facteur qui explique l'évolution des institutions est le mode de pensée d'une communauté économique qui est lui-même induit par l'évolution des modes d'appréhension du réel $^{27}$. Il n'y a donc pas de valeur positive ou négative attachée au concept d'institutions.

Dans l'approche institutionnelle, la question du conflit normatif est peu appréhendée car, par définition, les institutions, pour être efficaces, doivent être cohérentes. Sur une longue durée, et selon le mode de pensée évolutionniste, les institutions incohérentes entre elles ne peuvent que disparaître. Le conflit n'a donc de sens que pour un instant donné et il ne fait que traduire le lent ajustement des institutions aux évolutions environnementales et sociétales.

La théorie de la Tétranormalisation se différencie de la théorie institutionnelle ou néo-institutionnelle (North, 1981, 1990) car elle s'intéresse à la résolution du conflit normatif instantané. L'enjeu managérial, pour les organisations, réside dans la résolution du conflit aujourd'hui et maintenant, et non dans une perspective abstraite qui conduirait inéluctablement à la disparition des normes incompatibles entre elles et à l'émergence d'un nouvel ensemble normatif cohérent.

Même Oliver Williamson (1985) ne s'intéresse pas tant aux moyens organisationnels pour sortir d'une situation de conflit

\footnotetext{
${ }^{25}$ John Commons (1931, p.649) définit les institutions comme des actes collectifs permettant d'encadrer les actes individuels. Les droits et les devoirs, les coutumes et les lois, établissent le cadre dans lequel les actions individuelles peuvent se réaliser, soit en étant contraintes soit en étant au contraire facilitées.

${ }^{26}$ Les institutions sont donc le résultat de constructions humaines passées et elles sont en perpétuelle évolution du fait des pratiques actuelles qui viennent impacter les usages, les coutumes et les lois.

${ }^{27}$ Dans un processus qui s'autoalimente où les changements de pensée sont liés à l'évolution des modes de vie et aux évolutions technologiques.
} 
normatif qu'aux méthodes qui permettent d'éviter de se retrouver dans une situation insoluble de conflit normatif. Par exemple, son analyse des formes d'intégration explique pourquoi certaines entreprises privilégient l'intégration afin de bénéficier d'une réduction de leurs coûts de transaction. Le même raisonnement permet aussi d'expliquer, dans d'autres circonstances, le recours au marché, à la sous-traitance ou à l'externalisation d'activités afin d'abaisser les coûts de transaction. Dans cette approche, l'enjeu est de trouver la meilleure solution par rapport à un ensemble de paramètres qui déterminent l'environnement organisationnel. Rien n'est dit sur le management des situations organisationnelles où le dirigeant se trouve confronté à une situation non optimale. D'une certaine manière, la théorie des coûts de transactions permet d'expliquer pourquoi certaines décisions sont meilleures que d'autres d'un point de vue organisationnel, elle ne permet pas d'expliquer comment passer d'une situation à une autre, voire même comment sortir d'un piège où toutes les issues apparentes semblent bloquées (par exemple parce que recourir à de nouvelles formes institutionnelles de transaction supposerait la mobilisation de ressources dont l'organisation ne dispose pas). L'analyse des mécanismes de gouvernance (Williamson, 1996) se limite donc à la mise en évidence des meilleurs mécanismes de gouvernance mais appréhendés de façon statique : à telle situation correspond tel idéaltype de gouvernance.

Cette approche statique et idéaliste est dépassée par Masahiko Aoki (2010) car ce dernier, en s'appuyant sur les travaux d'Elinor Ostrom (1990), introduit explicitement non seulement la dynamique temporelle mais également les spécificités territoriales qui conditionnent l'existence de normes spécifiques locales. Dès lors, parce que chaque territoire a ses propres spécificités normatives, la notion d'idéal-type (Weber, 1920) se trouve reléguée non plus comme un idéal pratique à atteindre mais comme une forme de modélisation abstraite permettant de comprendre certains enchaînements de causalité.

Parce que l'idéal-type n'est jamais, et parce que chaque organisation se trouve confrontée à un environnement institutionnel (normatif) qui lui est particulier, l'analyse du conflit normatif prend toute son importance. N'étant pas dans la situation idéale où toutes les 
normes seraient cohérentes et convergentes, le dirigeant doit arbitrer en cherchant à tirer partie des différentes normes qui s'imposent à lui.

\subsection{Les théories psychiques}

Les questions économiques sur lesquelles les théoriciens se sont penchés au cours des deux derniers siècles reposaient sur le postulat de rareté des ressources. Parce que les ressources ne sont pas illimitées, comment assurer une répartition et une utilisation optimales des ressources? Le management des organisations visait donc à mettre en œuvre les processus permettant de trouver les solutions matérielles à la création de richesses en maximisant le résultat résiduel. Les prix et les contraintes techniques (les lois physiques de transformation de la matière) constituaient les éléments majeurs de ce management.

La place grandissante des normes dans les processus conduit à repenser le management en y introduisant un élément supplémentaire qui ne dépend pas des lois physiques. Les normes peuvent viser à une meilleure utilisation des ressources matérielles ; cependant, elles ne sont pas le résultat de phénomènes physiques mais de processus psychiques. Ce sont les êtres humains qui conçoivent, édictent et contrôlent les normes qui sont appliquées par les acteurs socio-économiques.

Le phénomène de Tétranormalisation, qui se traduit par la paralysie progressive des organisations confrontées à une diversité et à une pluralité de normes issues des quatre horizons, n'est donc pas un phénomène physique naturel mais un phénomène psychique qui n'a été que peu étudié jusqu'à présent.

La question fondamentale est : pourquoi l'être humain auraitil besoin de normes ? La réponse apportée par les religions est que la norme permet à l'être humain de vivre en société : tu ne tueras pas, tu ne voleras pas, etc. La norme permet à l'être humain de se confronter à des questions existentielles (des archétypes pour prendre le vocabulaire de Carl Gustav Jung) sans être immédiatement détruit ${ }^{28}$.

\footnotetext{
${ }^{28}$ Par exemple, pour Jung (1953), l'interdiction de l'inceste est une norme qui permet à l'enfant devenu adulte de se séparer de sa mère et donc à la fois de poursuivre son développement individuel et d'assurer le développement de l'espèce humaine.
} 
À notre époque moderne, la dimension existentielle de la norme a en grande partie disparu derrière des considérations techniques. Les normes comptables internationales ne prétendent pas explicitement régir le mode de relation des êtres humains. Néanmoins, derrière les choix techniques, se trouve une certaine conception de l'être humain et de sa vie en société ${ }^{29}$. Appréhender le conflit normatif qui se présente sous ses aspects techniques exige de pouvoir remonter aux racines du conflit et donc aux différentes représentations psychiques de l'être humain et de sa vie en société. Le conflit normatif n'est insoluble que parce qu'on en reste aux considérations techniques.

Par exemple, l'application des normes comptables internationales aux pays africains ne peut que soulever des conflits insolubles. Les normes comptables internationales reposent sur la référence à des prix du marché qui, implicitement, répondent aux critères de marchés actifs, c'est-à-dire concurrentiels et ouverts (Paper et Pigé, 2009). Or, dans de nombreux pays africains et pour un grand nombre de biens et services, les marchés ne présentent pas ces caractéristiques. Pour ne prendre que le marché du travail, l'équilibre de l'offre et de la demande pour la main d'œuvre non qualifiée conduit à l'établissement de prix de subsistance et non de prix d'équilibre ${ }^{30}$.

La résolution de ce conflit normatif sur un plan purement technique conduit à rechercher la mise en place d'institutions susceptibles de permettre l'établissement de véritables marchés. Ce faisant, on ignore les représentations culturelles qui donnent une primauté à la transaction sociale par rapport à la transaction économique. Ce conflit normatif renvoie à l'interaction entre les phénomènes économiques et les phénomènes sociaux. L'adage biblique «l'homme ne vit pas seulement de nourriture » reflète cette dimension sociale fondamentale. Dans l'interaction matérielle

${ }^{29}$ Cette conception était apparente dans les normes historiques IAS mais elle a progressivement disparu sous les considérations techniques dans les nouvelles normes IFRS.

${ }^{30}$ Le prix d'utilisation de la main d'œuvre non qualifiée est le prix qui permet à cette main d'œuvre de survivre. Il n'existe pas d'équilibre car l'offre de main d'œuvre est supérieure à la demande et, contrairement aux pays occidentaux, l'État n'intervient que très marginalement pour corriger les déficiences des marchés. 
d'échange de biens se joue également un échange social qui n'est pas nécessairement exprimé mais qui est pourtant fondamental (Malinowski, 1922 ; Mauss, 1923).

\section{3. - Le management de la Tétranormalisation}

Les normes portent sur des exigences à respecter et donc sur des comportements à adopter ou des pratiques techniques à mettre en œuvre. Le conflit normatif est initialement perçu comme un conflit entre des exigences contradictoires ou au minimum difficiles à concilier entre elles. La réponse naturelle est de tenter de concilier les exigences techniques en recherchant la solution technique qui permet de concilier toutes les exigences normatives et donc de faire disparaître le conflit normatif. Une solution alternative consiste à ignorer certaines exigences normatives en effectuant un arbitrage entre le coût du respect des normes et le coût pondéré anticipé du risque de sanction. Une dernière solution existe qui consiste à repenser le conflit normatif dans un cadre territorial et dynamique.

\subsection{Le respect des contraintes normatives}

Deux approches sont habituelles, celle qui part des spécialistes des différentes normes et celle qui part du sommet de l'organisation. La première approche est susceptible de conduire à une perte d'innovation en sus des coûts élevés générés par le recours aux experts. La seconde approche exige une pluralité de ressources inaccessibles aux petites et moyennes organisations.

La norme touche à des enjeux techniques concrets (une certaine façon de faire). Il est donc logique de s'en remettre aux spécialistes, ceux qui savent, les experts de la norme ou du domaine normatif concerné (les comptables pour les normes comptables, les avocats pour l'application des contrats, les gestionnaires de ressources humaines pour le droit du travail, les financiers pour les normes prudentielles ou les normes d'information financière, etc.).

Le constat de la Tétranormalisation est que cette délégation de la responsabilité aux experts finit par rencontrer des nœuds insolubles quand les experts se trouvent en conflit les uns avec les autres sur des objets pour lesquels leurs normes de référence 
préconisent ou imposent des solutions incompatibles. Une démarche consiste à réunir les experts pour identifier les champs du possible compte tenu des multiples contraintes normatives. Cette solution, mise en œuvre dans les organisations disposant des ressources suffisantes, s'apparente aux groupes de projet dans l'industrie (en particulier automobile). Des experts de domaine différents (achat, design, conception de la fabrication, marketing, finance, etc.) sont réunis dans une équipe projet pour identifier en amont, et de façon concertée, les difficultés à résoudre et les solutions techniques qui permettent de répondre aux attentes des différents acteurs (Aoki et Dore, 1994 ; Liker et Hoseus, 2008). Une telle solution est difficile à mettre en œuvre dans les petites organisations, car elle nécessite des ressources humaines significatives et correspond à un lourd investissement.

Cependant, le nœud de Tétranormalisation le plus pénalisant n'est pas dans le coût des ressources à mettre en œuvre (il s'agit de coûts visibles, mesurables) mais dans les solutions qui finissent par s'imposer et qui incluent de nombreux coûts cachés (Savall et Zardet, 2015). En effet, le consensus à partir des visions d'experts conduit à un nombre limité d'options et entrave l'innovation. Parce que l'innovation comporte des risques significatifs qui ne sont pas totalement contrôlables (identifiables) ex ante (et qui n'offrent donc pas la garantie de la conformité), les experts vont généralement privilégier les solutions techniques éprouvées garantes de la conformité recherchée. Par conséquent, les solutions techniques mises en œuvre par différentes organisations œuvrant dans un même secteur d'activité vont tendre à être uniformes, non pas tant en raison d'une similitude des personnes impliquées dans ces projets en tant qu'experts mais en raison du processus lui-même. Parce que chaque expert est légitimé par le champ normatif auquel il fait référence, il lui est difficile de s'engager dans des solutions qui le mettraient en porteà-faux (en danger) vis-à-vis du respect (isomorphique : DiMaggio et Powell, 1983) de ces normes.

La Tétranormalisation ne conduit donc pas nécessairement à la paralysie, cela dépend des ressources humaines déployées pour sortir des conflits normatifs, mais elle conduit nécessairement à une forme d'isomorphisme où les objets, les fonctions et les processus tendent à se standardiser à outrance. In fine, c'est la question de la 
diversité qui est en jeu, y compris quand on applique des normes de développement durable censées permettre la conservation de la diversité.

Si l'approche par les experts conduit à un isomorphisme de fait, l'approche par le haut semble être un moyen de réintroduire une diversité de pratiques. En effet, alors que le consensus peut conduire à un isomorphisme réducteur, l'instinct (la libération d'une forme de perception inconsciente des enjeux organisationnels) du décideur peut réintroduire la diversité. Cet instinct entrepreneurial est néanmoins complexe car, par lui-même, il ne garantit ni l'atteinte des objectifs ni le respect de la conformité. Une approche visionnaire par le dirigeant nécessite donc de combiner à la fois une compréhension rationnelle des contraintes normatives et une ouverture à l'innovation. Un cas emblématique de cette vision est le lancement de la Toyota Prius en 1997 au Japon et en 2000 aux Etats-Unis à une époque où le prix du pétrole (moins de $30 \$$ le baril en moyenne en 2000) favorisait les gros véhicules non électriques.

Cette approche par le haut nécessite paradoxalement des moyens humains très importants. En effet, si le dirigeant veut concilier vision et respect des contraintes normatives, il est obligé d'imposer un cahier des charges très contraignant aux experts techniques. Sauf à se mettre en contravention avec les normes, la solution par le haut implique une mobilisation considérable de ressources qui en réduit l'utilité à un nombre restreint d'organisations ou à des projets suffisamment significatifs pour justifier le déploiement des ressources nécessaires.

\subsection{L'arbitrage sur les coûts de la conformité}

Confrontée aux coûts engendrés par la résolution des conflits, une organisation peut décider de s'affranchir de tout ou partie des contraintes normatives. Ce faisant, l'organisation s'expose à des sanctions. Dans une approche purement économique, le choix des normes à respecter s'impose par une analyse des coûts engendrés par la conformité, comparés au coût des sanctions en cas de contrôle. Ce dernier coût est fonction du montant des pénalités encourues et de la probabilité d'être contrôlé et sanctionné.

Cette approche économique comporte néanmoins de nombreuses difficultés. Tout d'abord elle néglige la dimension 
sociétale du comportement de l'organisation. Ne pas être conforme peut entraîner des coûts cachés liés à une dégradation de l'image de marque de l'organisation, à une démotivation de ses employés, à une détérioration des relations contractuelles avec les fournisseurs et les sous-traitants qui mettent en doute la valeur des engagements donnés par l'organisation, etc.

Ensuite, il existe une grande incertitude sur la probabilité d'être contrôlé et sur le montant des sanctions encourues. Dans certains environnements institutionnels, les sanctions peuvent faire l'objet de négociations et, généralement, c'est le rapport de force entre l'organisation et l'organe de contrôle qui sera déterminant ${ }^{31}$. Dans la mesure où la sanction a lieu dans le futur avec une probabilité d'occurrence, se pose la question de l'actualisation de la valeur de la sanction. Si l'acteur présente une très forte aversion au risque, une sanction faible et une probabilité de contrôle faible pourront suffire à assurer la conformité. Si, au contraire, l'acteur présente une appétence au risque, seules des sanctions très importantes et des contrôles fréquents pourront assurer la conformité ${ }^{32}$.

L'arbitrage économique sur les coûts de la non-conformité pose un dernier problème qui est celui des ressources à engager pour évaluer le coût des alternatives. Il est vraisemblable que de petites organisations ne disposent pas des ressources nécessaires à cette analyse économique et que, par conséquent, la résolution du conflit de Tétranormalisation reposera sur des facteurs cognitifs liés aux différentes valeurs éthiques que le dirigeant pourra attribuer aux diverses sources de normalisation.

\section{3. $L$ 'approche territoriale dynamique}

Les conflits normatifs se dévoilent habituellement sous leurs aspects techniques. Ce sont des contraintes concrètes, précises qui rendent difficile l'application simultanée d'une pluralité de normes

${ }^{31}$ Une non-conformité commise par un acteur puissant peut aboutir à une sanction faible si l'organisme de contrôle se trouve démuni mais, inversement, cette nonconformité peut conduire à une sanction plus sévère si l'organisme de contrôle cherche à faire un exemple.

${ }^{32}$ C'est notamment le cas pour les normes touchant au blanchiment d'argent ou au financement du terrorisme. 
d'origines distinctes. D'une certaine manière, la Tétranormalisation résulte de la rencontre de normes abstraites ${ }^{33}$ avec un milieu concret. Et c'est dans ce milieu concret que se matérialisent les conflits.

Rechercher un mode de résolution des conflits de Tétranormalisation suppose donc de prendre en compte les spécificités du milieu concret. L'approche territoriale permet d'introduire l'incarnation des acteurs. Les conflits normatifs existent parce qu'in fine ce sont des êtres humains qui doivent composer avec les normes qui leur sont imposées. Le territoire permet d'appréhender la réalité des contraintes multiples auxquelles les acteurs sont confrontés. Or ce sont ces contraintes (géographiques, climatiques, environnementales, familiales, religieuses, sociales) qui vont donner une acuité plus ou moins grande aux conflits normatifs. À titre d'exemple, l'application des normes comptables internationales ne générera pas les mêmes conflits normatifs en Europe et en Afrique, au Maroc et au Cameroun. En effet, les modes de transaction, l'existence de marchés centralisés, la dématérialisation des flux financiers, la présence de multinationales, la nature des emplois, etc. vont façonner concrètement des notions abstraites comme le prix du marché ou le taux d'actualisation.

Comprendre le territoire où se déroule un conflit normatif c'est pouvoir comprendre le jeu des acteurs et les logiques mises en œuvre pour défendre des intérêts particuliers sous couvert de conformité aux diverses normes. Cependant, cette démarche n'est pas suffisante, elle doit s'accompagner d'une inscription dans la durée. À un instant donné, une situation conflictuelle semble insoluble parce qu'elle est perçue comme un équilibre, défavorable à l'intérêt général, en raison des forces qui s'opposent. Replacer le conflit normatif dans la durée permet à la fois de comprendre la dynamique qui anime les acteurs $^{34}$ et de percevoir la dynamique qui anime les conflits normatifs. En effet, certains conflits normatifs ne sont dus qu'à une conjonction particulière et ponctuelle de divers phénomènes. Attendre

${ }^{33}$ Les normes ont nécessairement un caractère d'abstraction pour pouvoir être applicables de façon générale. Même quand des cas particuliers sont prévus, ces derniers conservent toujours une part d'abstraction. Autrement, il s'agirait d'une collection de cas particuliers et la norme disparaîtrait d'elle-même.

${ }^{34}$ Sur une longue période certains acteurs pourront estimer avoir beaucoup perdu et ils pourront être susceptibles d'adopter des attitudes intransigeantes. 
que les phénomènes évoluent (ou hâter leur évolution) est parfois la meilleure solution pour favoriser la résolution d'un conflit normatif. Inversement, l'observation de la dynamique normative peut aboutir au constat que les conflits normatifs ne peuvent que s'aggraver. Par conséquent, la solution au conflit normatif n'est peut-être pas tant au niveau local qu'au niveau normatif et la réponse peut alors être apportée en fédérant les acteurs concernés par ce conflit normatif.

Mais la dynamique n'est pas seulement celle du cadre institutionnel et normatif. Elle est également le fait de l'organisation. En se mettant en mouvement, en modifiant ses processus et en faisant évoluer ses frontières, l'organisation peut réagir aux conflits normatifs, en s'abstrayant du champ de certains conflits pour gagner des environnements où les espaces de liberté sont plus importants et où les normes, au lieu de contraindre le jeu des acteurs, contribuent à leur développement. La dynamique permet de considérer les conflits normatifs comme des cadres contraignants qu'on ne peut dépasser qu'en donnant du mouvement à l'organisation. Sur un plan statique, les conflits normatifs sont mortifères. Ils sont au contraire sources de dynamisme si l'organisation arrive à les utiliser comme une force externe de changement. La normalisation liée au développement durable et à la responsabilité sociale et environnementale en est une bonne illustration.

\section{4. - Conclusion}

La Tétranormalisation est initialement apparue comme le constat des contraintes normatives désordonnées auxquelles les dirigeants d'organisations étaient confrontés. Le désordre normatif ne résulte pas d'une incompétence des normalisateurs mais d'une multiplication des normalisateurs. Si chaque organe de normalisation poursuit une cohérence qui lui est propre, l'effacement relatif de l'État-nation a fait disparaître l'organe normalisateur supposé assurer la cohésion entre des normalisations multiples et diverses. À une logique normative pyramidale et hiérarchique s'est substituée une logique imbricationnelle (chaque ensemble normatif cherche sa place dans le vaste écheveau qui gère les relations économiques et sociales). 
Ce constat initial s'appuyait sur une approche socioéconomique visant à mesurer les coûts cachés organisationnels générés par les dysfonctionnements dans la conduite des activités et des processus. Selon cette approche, l'efficience économique n'est pas atteinte (et parfois n'est même pas poursuivie) parce que les interactions sociales ne sont pas prises en compte. En mesurant l'impact économique des dysfonctionnements, il devient alors possible d'agir sur les relations sociales afin d'optimiser les processus et contribuer à une plus grande création de valeur économique.

La mise en relation de la Tétranormalisation avec les théories institutionnelles et néo-institutionnelles permet de rajouter une troisième dimension fondamentale qui est la dimension psychologique. En effet, la Tétranormalisation est le constat de l'enfermement normatif technique. C'est la normalisation des solutions techniques qui conduit à interdire toute forme d'innovation et qui conduit les dirigeants à ne gérer que des contraintes (ou à concevoir des stratégies complexes pour s'en affranchir ${ }^{35}$ ). Une réponse purement technique n'est concevable qu'en disposant de ressources considérables pour identifier les espaces de liberté résiduels. L'intégration de la dimension psychique vient bouleverser ce cadre rigide en replaçant les techniques à leur place et en obligeant les acteurs à se situer non plus dans le cadre d'un exercice d'optimisation avec des contraintes rigides et immuables mais au contraire dans un cadre évolutif où l'évolution est générée par les acteurs eux-mêmes. C'est parce que les acteurs prennent conscience des possibilités ouvertes par un changement de référentiel, qu'il devient possible de dépasser le conflit normatif et d'ouvrir de nouveaux champs d'innovation. La théorie de la Tétranormalisation est donc une théorie psycho-socio-économique qui permet de poursuivre l'œuvre théorique de Thorstein Veblen (1898) en se focalisant sur la dimension normative qui unit les actions individuelles et collectives (Commons, 1931).

${ }^{35}$ L'évasion fiscale (autrement dénommée optimisation fiscale) des grands groupes internationaux et le recours aux paradis fiscaux constituent des moyens de contournement des contraintes normatives. 


\section{RÉFÉRENCES BIBLIOGRAPHIQUES}

AOKI M., Corporations in Evolving Diversity: Cognition, Governance and Institutions, Oxford University Press, 2010.

AOKi M. et Dore R. éd., The Japanese Firm, Clarendon paperbacks, 1994.

BESSIRE D., "Comptabilité financière, management des risques bancaires et gouvernance d'entreprise: la colonisation par les normes », in Bessire et al., Normes: Origines et Conséquences des Crises, Economica, 2010, p.55-62.

Bessire D., Cappelletti L. et Pige B. éd., Normes: Origines et Conséquences des Crises, Economica, 2010.

BRANElleC G. et CADET I., «La norme ISO 26000 : nouveau fil d'Ariane pour les organisations confrontées à la Tétranormalisation? Le cas d'Armor Lux ", in Cappelletti et al., Dynamique normative, Arbitrer et négocier la place de la norme dans l'organisation, EMS, 2015, p.61-72.

CADET I., «Les droits de l'homme dans l'Union européenne : un modèle de normes universelles?», in Bessire et al., Normes: Origines et Conséquences des Crises, Economica, 2010, p.149-162.

Cappelletti L., Pige B. et Zardet V. éd., Dynamique normative, Arbitrer et négocier la place de la norme dans l'organisation, EMS, 2015.

Commons J.R., "Institutional Economics", American Economic Review, 21(4), 1931, p.648-657.

Cyert R.M. et MARch J.G., A Behavioral Theory of the Firm, Blackwell, éd. 1992, 1963.

DiMaggio P. et Powell W.W., "The iron cage revisited: Collective rationality and institutional isomorphism in organizational fields", American sociological review, 48(2), 1983, p.147-160.

DuPUY Y. et NARO G., « La Tétranormalisation et l'hôpital public : pour une interprétation organisationnelle », in Cappelletti et al., Dynamique normative, Arbitrer et négocier la place de la norme dans l'organisation, EMS, 2015, p.189-202.

Goffman E., Stigma: Notes on the management of spoiled identity, Simon and Schuster, 1963.

Hirschman A. O., Exit, Voice, and Loyalty, Harvard University Press, 1970.

Jung C.G., Métamorphoses de l'âme et de ses symboles, Georg, 1953.

LEwis D., Convention: A philosophical study, Harvard University Press, 1969.

LiKer J.K. et Hoseus M., Toyota culture, Mc Graw Hill, 2008.

MalinowsKi B., Argonauts of the Western Pacific, Routledge, éd. 2014, 1922.

MARechal J-P., "L’héritage négligé de François Perroux", Économie politique, octobre, 2003, p.47-63. 
MAuss M., «Essai sur le don, forme et raison de l'échange dans les sociétés archaïques », in Sociologie et anthropologie, PUF, éd. 2010, 1923.

Mounier E., InYesto J.F. et PALAcios J.M., Le personnalisme, PUF, 1950.

NoRTH D.C., Structure and Change in Economic History, Norton, 1981.

NorTH D.C., Institutions, Institutional Change and Economic Performance. Cambridge University Press, 1990.

Ostrom E., Governing the Commons - The evolution of institutions for collective action, Cambridge University Press, 1990.

PAPER X. et PIGE B., Normes comptables internationales et gouvernance des entreprises : le sens des normes IFRS, EMS, 2009.

PERON M., «Approche lexicographique de la notion de norme et de son évaluation historique ", in Bessire et al., Normes: Origines et Conséquences des Crises, Economica, 2010, p.13-22.

Perroux F., Pouvoir et économie, Bordas, 1973.

RUTHERFORD M., "J.R. Commons's Institutional Economics", Journal of Economic Issues, 17(3), 1983, p.721-744.

SAVAll $H$. et ZARDET V., Tétranormalisation, défis et dynamique, Economica, 2005.

SAVAll H. et ZARDET V., Maîtriser les coûts et les performances cachés, Economica, 2015.

Simon H. A., Administrative Behavior, The Free Press, éd. 1997, 1945.

TCHOTOURIAN I., «L'entreprise surencadrée : l'illustration de la rémunération des dirigeants d'entreprise », in Bessire et al., Normes : Origines et Conséquences des Crises, Economica, 2010, p.71-78.

THIBIERGE C. éd., La force normative, naissance d'un concept, LGDJ, 2009.

ThiBIERGE C. éd, La densification normative, découverte d'un processus, Mare \& Martin, 2014.

VEBLEN T., "Why is Economics not an evolutionary science?", Quarterly Journal of Economics, 12(4), 1898, p.373-397.

Weber M., "Wirtschaft und Gesellschaft", édité par Parsons T., The Theory of Social and Economic Organization, The Free Press, éd. 1947, 1920.

Williamson O.E., The Economic Institutions of Capitalism, The Free Press, 1985.

Williamson O.E., The mechanisms of governance, Oxford University Press, 1996. 